\title{
Modeling and Classification of the ST Segment Morphology for Enhanced Detection of Acute Myocardial Infarction
}

\author{
Reza Firoozabadi, Richard E Gregg, Saeed Babaeizadeh
}

\author{
Advanced Algorithm Research Center, Philips Healthcare, Andover, MA, USA
}

\begin{abstract}
A number of cardiac conditions such as acute pericarditis $(P C)$ and early repolarization (ER) cause $S T$ elevation which mimics ST-segment Elevation Myocardial Infarction (STEMI). Current guidelines recommend analyzing ST segment morphology to distinguish STEMI from these confounders. ST elevation in PC and ER (and possibly in STEMI) is concave (upward) in the JTpeak interval, while a convex or straight ECG ST segment is associated with the diagnosis of STEMI. We developed an algorithm to classify concavity characteristic of the ST segment. A quadratic polynomial regression algorithm was introduced to model the shape of JTpeak interval. Our diagnostic algorithm generated representative beats and measured the fiducial points and standard measurements such as ST level in 12-lead 10-sec segments of ECG recordings. JTpeak interval was modeled by a parabola using a least-squares polynomial regression algorithm. Classifier features such as curvature, parabola direction and vertex, model fit error, and the noise measure were determined. A bootstrap-aggregated tree ensemble classifier determined the ST segment shape. Our algorithm was evaluated on a 12-lead ECG database collected in two medical centers. Our ST segment polynomial regression model exhibited significant improvement in concavity detection versus a simple conventional method.
\end{abstract}

\section{Introduction}

Some cardiac diseases such as pericarditis (PC), early repolarization (ER), left bundle branch block (LBBB), and left ventricular hypertrophy (LVH) show ST elevation, confounding the diagnosis of ST-segment Elevation Myocardial Infarction (STEMI). Extra features, such as ST segment morphology, are required in addition to ST elevation, to distinguish STEMI from ST elevation confounders. Pericarditis and early repolarization show concave (upward) ST elevation against STEMI with convex or straight-line ST segment (except anterior STEMI). While LBBB and LVH have typically concave ST elevation, we focus only on PC and ER confounders.
Each cardiac condition has a set of specific criteria [14]. The set of criteria for diagnosis of pericarditis includes widespread concave (upward) ST elevation with PRsegment depression in some leads [5]. Early repolarization is diagnosed by concave upward ST elevation mostly in pre-cordial leads and possibly extended to the other leads without reciprocal ST depression, and presence of J-waves or J-point notches/slurs [6]. Ratio of T-wave amplitude to ST level is a major discriminator between early repolarization (high) and pericarditis (low). STEMI is defined by ST elevation (typically convex or straight, but could be concave in a large fraction of anterior cases) in at least two contiguous leads, ST depression in reciprocal leads, presence of Q-waves, and wide upright or inverted T-waves $[7,8]$.

Most conventional algorithms determine concavity by simple measures such as the area between the JTpeak interval and the line connecting its ends or the position of the point in the JTpeak interval with maximum distance from the straight line. However, these simple models behave poorly in the presence of artifact. We modeled the ST segment morphology by a low-dimensional polynomial and defined several features to classify the ST segment concavity. The criteria to diagnose STEMI or its confounders make use of the concavity feature in addition to the other features specific to each cardiac condition.

The rest of this paper is organized as follows. Section 2 describes the method and material including the algorithm overview, polynomial regression model, classifier features, database, and classifier description. Results are provided in Section 3. Discussion and conclusions are presented in Section 4.

\section{Method and Material}

\subsection{Algorithm Overview}

Block diagram of our algorithm is shown in Figure 1. Our algorithm reads the multi-lead data (12-lead or more) and analyzes them in non-overlapping 10-second segments. The algorithm generates a representative beat in each segment, identifies the fiducial points, finds standard measurements such as ST level. 
We determine JTpeak interval which is the interval between the end of QRS complex (J-point) and the peak of the T-wave. This interval is modeled by a fitted section of the quadratic curve (parabola) using a least-squares polynomial regression approach. The classifier features such as the parabola opening direction and its vertex location, polynomial model fit error, noise measure, and curvature measures are determined from the fitted curve and the original JTpeak interval. These features determine whether the curve fits the JTpeak properly and if it is concave upward by using a classifier.
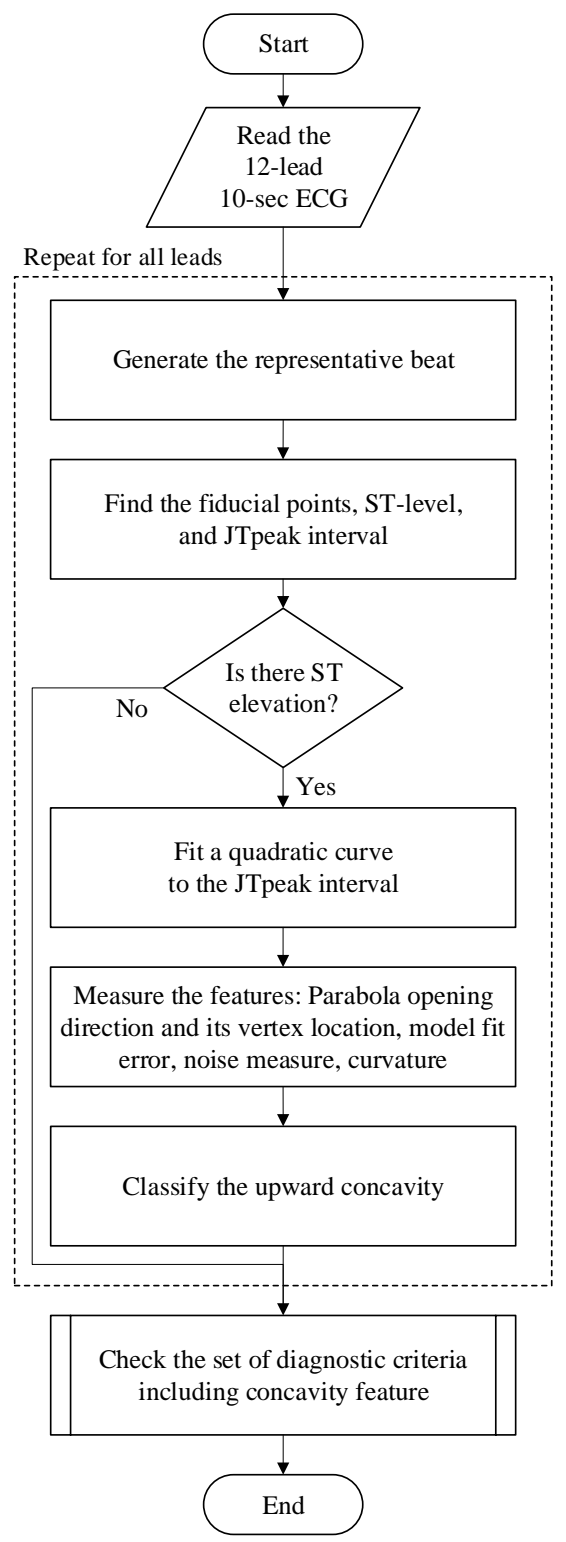

Figure 1. Block diagram of the algorithm. This procedure is repeated for all leads in the data.

Concavity is one of the features used in the set of diagnostic criteria for each of the cardiac conditions including STEMI, pericarditis, or early repolarization.

\subsection{Polynomial Regression model}

Using quadratic polynomial regression analysis, JTpeak interval was modeled by a low-dimensional curve (parabola) defined by only three coefficients $\mathrm{a}, \mathrm{b}$, and c:

$$
\hat{y}=a x^{2}+b x+c
$$

To explicitly derive these coefficients, we used leastsquares method to minimize the error between the model and the JTpeak interval regarding margins at its both ends to avoid the roundness around J-point and T-wave peak. The regression error is the sum of the squared errors between the model $(\hat{y})$ and the original signal $(y)$ at each sample $k$ over $N$ samples $\left(x_{k}, y_{k}\right), k=1, \ldots, N$ :

$$
S=\sum_{k}\left(e_{k}\right)^{2}=\sum_{k}\left(y_{k}-\left(a x_{k}{ }^{2}+b x_{k}+c\right)\right)^{2}
$$

which is minimized by:

$$
\frac{\partial S}{\partial a}=0, \frac{\partial S}{\partial b}=0, \frac{\partial S}{\partial c}=0
$$

These derivatives define a system of three equations which is solved for the three unknown coefficients using the Cramer's rule:

$$
a=\frac{\Delta_{a}}{\Delta}, \quad b=\frac{\Delta_{b}}{\Delta}, \quad c=\frac{\Delta_{c}}{\Delta}
$$

The determinants $\left(\Delta^{\prime} \mathrm{s}\right)$ are defined in closed forms in terms of $x_{k}$ and $y_{k}, k=1, \ldots, N$.

Figure 2 displays an example of the quadratic polynomial regression of the JTpeak interval in a representative beat extracted from a 10-second ECG segment. The regression parabola, osculating circle with the curvature radius, and the line connecting both ends of the JTpeak interval are illustrated.

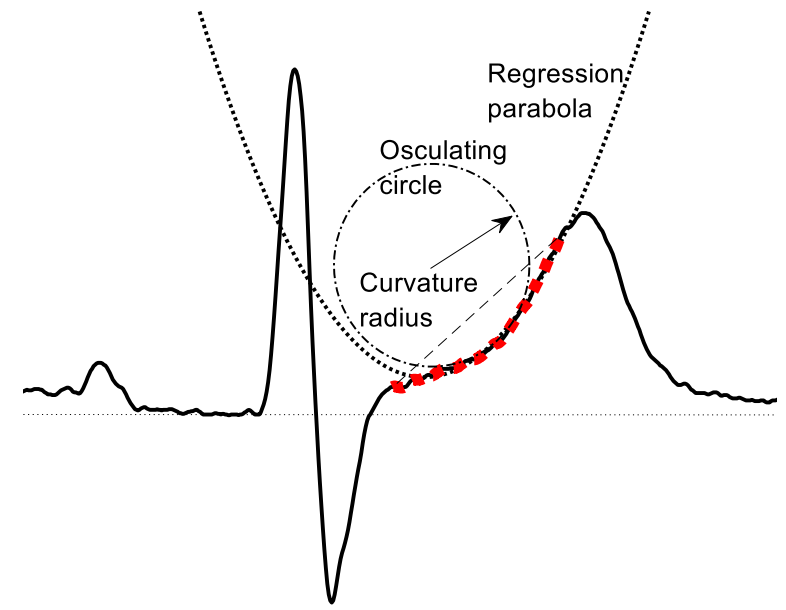

Figure 2. Example of quadratic polynomial regression of the JTpeak interval (large dots). A section of the regression parabola fits the JTpeak interval and the curvature at each point of the JTpeak interval is defined by a curvature radius of an osculating circle at that point. 


\subsection{Classifier Features}

Other than the coefficient of the quadratic term in the hyperbola equation $(a)$ which determines the opening direction of the hyperbola, we take into account other features which determine the position of parabola section fitting the JTpeak interval, goodness of fit, signal quality, and the JTpeak interval degree of curve.

Location of the vertex of parabola determines the shape of parabola section fitting the JTpeak interval:

$$
v=-\frac{b}{2 a}
$$

Model fit error describes the difference between the fitted curve and the smoothed JTpeak interval and is presented by the coefficient of determination (R-squared):

$$
R^{2}=1-\frac{\sum(\tilde{y}-\hat{y})^{2}}{\sum(\tilde{y}-\tilde{y} \text { ave })^{2}}
$$

where $\tilde{y}$ is the JTpeak interval smoothed by a moving average filter and $\tilde{y}_{a v e}$ is its average.

Noise measure is the mean difference of the ECG values in the JTpeak interval from their smoothed values normalized by the range of the JTpeak interval:

$$
N M=\frac{\sum|y-\tilde{y}|}{N \cdot\left|\tilde{y}_{\max }-\tilde{y}_{\min }\right|}
$$

Degree of curve in the JTpeak interval is determined by two features. First feature is $\hat{\kappa}_{\text {max }}$, defined by the scaled maximum curvature:

$$
\hat{\kappa}_{\text {max }}=\max (\kappa) \cdot\left|\hat{y}_{\text {max }}-\hat{y}_{\text {min }}\right|
$$

where curvature $\kappa$ (the measure which determines the degree to which the fitted model is curved) is presented by:

$$
\kappa=\frac{\left|\hat{y}(x)^{\prime \prime}\right|}{\left(1+\hat{y}(x)^{\prime 2}\right)^{\frac{3}{2}}}
$$

Second curvature feature is defined based on the curvature variation during the JTpeak interval:

$$
\kappa_{\text {ratio }}=\frac{\max (\kappa)}{\min (\kappa)}
$$

\subsection{Database}

The algorithm was developed on a subset of 12-lead ECG data selected from databases collected in two medical centers. We selected a total of 300,10 -sec segments of 12 lead ECG records annotated either by experts' reviews or from discharge charts which include STEMI $(\mathrm{n}=100)$, PC $(n=100)$, and ER $(n=100)$.

All leads were manually reviewed and annotated with the binary decision of visible upward concavity or not. We selected the leads which showed ST elevation greater than $50 \mu \mathrm{V}$. As a result, 1,820 concavity-annotated leads were chosen with one of those three cardiac conditions. These concavity annotations were used as the reference in our classification.

\subsection{Classifier Description}

We developed a bootstrap-aggregated ensemble of decision trees to classify the ST segment concavity. The classifier consists of 100 trees with maximum 100 splits and takes the six described classifier features (coefficient $a, R^{2}, N M, v, \hat{\kappa}_{\text {max }}$, and $\kappa_{\text {ratio }}$ ) measured from 1,820 leads with ST elevation as predictors. The output is binary with the positive event being the concave upward ST segment.

\section{Results}

Table 1 summarizes the performance of our regression algorithm using a 10 -fold cross validation. Also listed for comparison is the performance of a simple conventional method that determines the concavity of ST segment by position of the point on JTpeak interval maximizing the distance to the line connecting the JTpeak interval boundaries, whether it is located above or below the line.

Figure 4 shows the confusion matrix of the outcome. Figure 5 displays the classifier ROC with $\mathrm{AUC}=97 \%$. Figure 6. Illustrates the example of 12-lead ECG representative beats and the fitted parabolas for ST segments with at least $50 \mu \mathrm{V}$ elevation in patients with pericarditis, early repolarization, or STEMI.

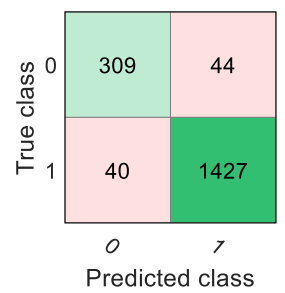

Figure 4. Confusion matrix of the classifier.

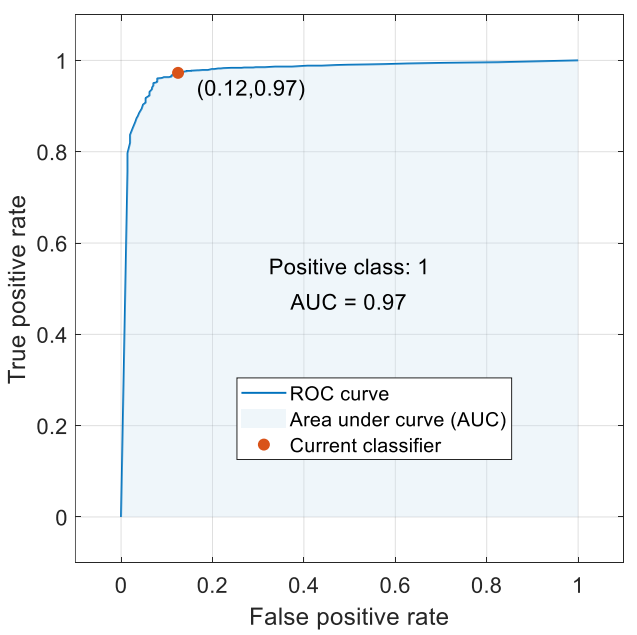

Figure 5. Classifier outcome ROC with AUC = 0.97. 

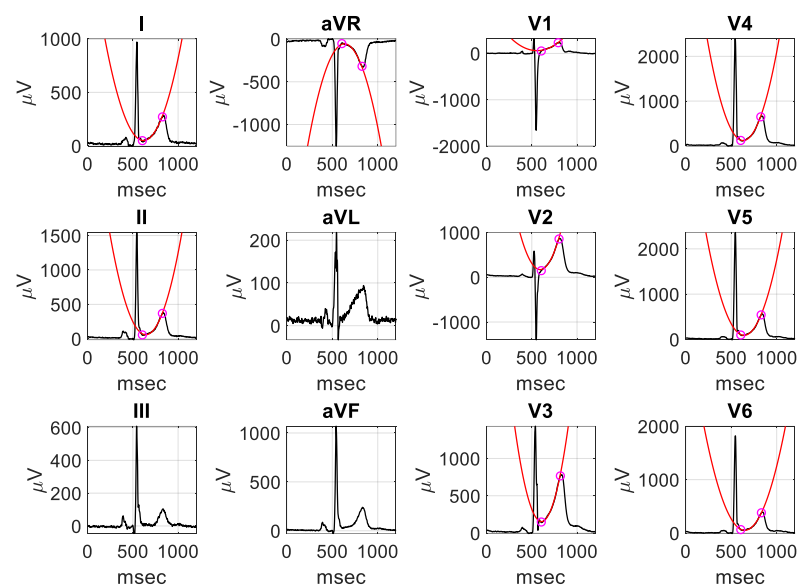

(a) Pericarditis
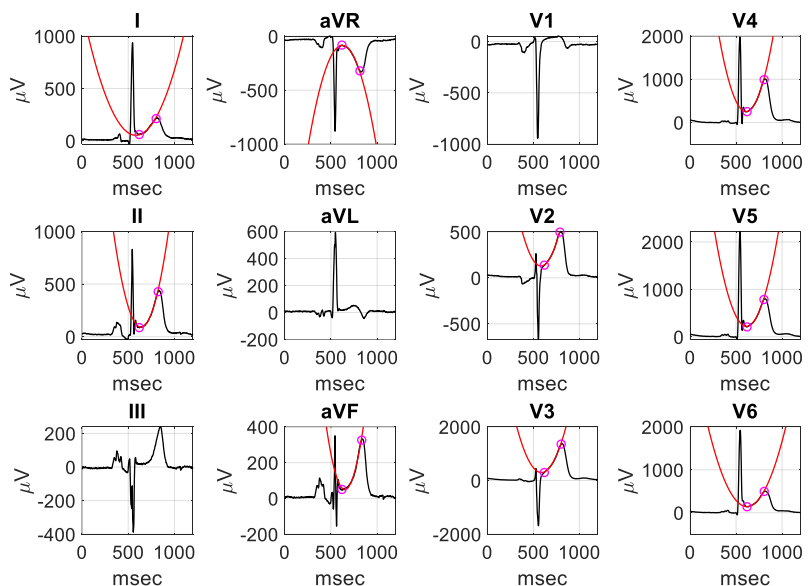

(b) Early repolarization
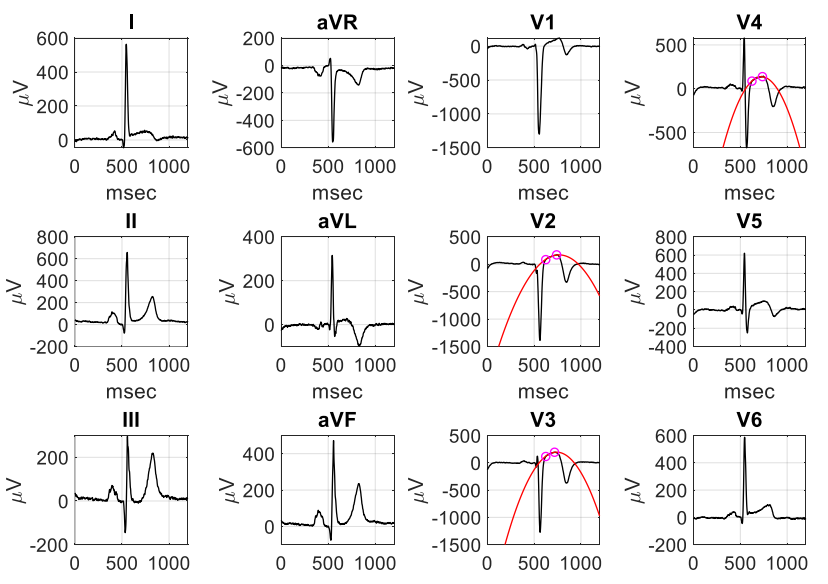

(c) STEMI

Figure 6. Example of 12-lead ECG representative beats in patients with (a) pericarditis, (b) early repolarization, and (c) STEMI. All ST segments with at least $50 \mu \mathrm{V}$ elevation are fitted to a section of parabola. Note that the lead aVR displays inverted ST segment and hence a reverse concavity direction.
Table 1. Regression algorithm performance compared to a simple method

\begin{tabular}{lcccc}
\hline Algorithm & $\mathrm{Se}(\%)$ & $\mathrm{Sp}(\%)$ & $\mathrm{PPV}(\%)$ & $\mathrm{NPV}(\%)$ \\
\hline Polynomial & 97 & 88 & 97 & 89 \\
\hline Simple & 96 & 58 & 91 & 77 \\
\hline
\end{tabular}

\section{Discussion and Conclusions}

Modeling the ST segment by a polynomial regression model showed significant improvement versus a simple conventional method of concavity detection. The model is low-dimensional, is defined by only three parameters, and is solved using closed form expressions, which makes it computationally efficient and a proper candidate for easy implementation in systems with limited power, memory or processing performance. False cases are mostly due to the borderline concavity, inaccuracies in the JTpeak determination, and high nonlinearity in the curvature equation. Using a larger validation database will enhance the algorithm performance evaluation.

\section{References}

[1] Hanna EB, Glancy DL. ST-segment elevation: Differential diagnosis, caveats. Clin J Med. 2015 Jun;82(6):373-84.

[2] Brady WJ, Syverud SA, et al. Electrocardiographic STsegment Elevation: The Diagnosis of Acute Myocardial Infarction by Morphologic Analysis of the ST Segment. Acad Emerg Med. 2001 Oct;8(10):961-7.

[3] Birnbaum Y, Nikus K, et al. The Role of the ECG in Diagnosis, Risk Estimation, and Catheterization Laboratory Activation in Patients with Acute Coronary Syndromes: A Consensus Document. Annals of Noninvasive Electrocardiology. 2014;19(5):412-25.

[4] Gregg RE, Babaeizadeh S. Improving automatic detection of acute myocardial infarction in the presence of confounders. CinC 2014: 637-40.

[5] Ariyarajah V, Spodick DH. Acute pericarditis: Diagnostic cues and common electrocardiographic manifestations. Cardiol in Review 2007;15:24-30.

[6] Macfarlane PW, Clark EN. ECG measurements in end QRS notching and slurring. J Electrocardiol 2013;46:385-9.

[7] O'Gara PT, Kushner FG, Ascheim DD, et al. 2013 ACCF/AHA Guideline for the management of ST-elevation myocardial infarction: A report of the American College of Cardiology Foundation/American Heart Association Task Force on practice guidelines. Circulation 2013;127:e362e425.

[8] Steg PG, James SK, Atar D, et al. ESC Guidelines for the management of acute myocardial infarction in patients presenting with ST-segment elevation. Eur Heart J 2012;33:2569-619.

Address for correspondence.

Reza Firoozabadi

Philips Healthcare

3000 Minuteman Rd, Andover, MA 01810

reza.firoozabadi@philips.com 